\title{
Brain Tumor Detection And Localization In Magnetic Resonance Imaging
}

\author{
Ed-Edily Mohd. Azhari ${ }^{1}$, Muhd. Mudzakkir Mohd. Hatta ${ }^{1}$, Zaw Zaw Htike ${ }^{1 *}$ and \\ Shoon Lei Win ${ }^{2}$ \\ ${ }^{1}$ Department of Mechatronics Engineering, IIUM, Kuala Lumpur, Malaysia \\ ${ }^{2}$ Department of Biotechnology Engineering, IIUM, Kuala Lumpur Malaysia
}

\begin{abstract}
A tumor also known as neoplasm is a growth in the abnormal tissue which can be differentiated from the surrounding tissue by its structure. A tumor may lead to cancer, which is a major leading cause of death and responsible for around $13 \%$ of all deaths world-wide. Cancer incidence rate is growing at an alarming rate in the world. Great knowledge and experience on radiology are required for accurate tumor detection in medical imaging. Automation of tumor detection is required because there might be a shortage of skilled radiologists at a time of great need. We propose an automatic brain tumor detection and localization framework that can detect and localize brain tumor in magnetic resonance imaging. The proposed brain tumor detection and localization framework comprises five steps: image acquisition, pre-processing, edge detection, modified histogram clustering and morphological operations. After morphological operations, tumors appear as pure white color on pure black backgrounds. We used 50 neuroimages to optimize our system and 100 out-of-sample neuroimages to test our system. The proposed tumor detection and localization system was found to be able to accurately detect and localize brain tumor in magnetic resonance imaging. The preliminary results demonstrate how a simple machine learning classifier with a set of simple image-based features can result in high classification accuracy. The preliminary results also demonstrate the efficacy and efficiency of our five-step brain tumor detection and localization approach and motivate us to extend this framework to detect and localize a variety of other types of tumors in other types of medical imagery.
\end{abstract}

\section{KEYWORDS}

Tumor Detection, Medical Imaging, Computer Vision, Machine Learnin .

\section{INTRODUCTION}

Based on statistics, tumors are the second cause of cancer-related deaths in children (both males and females) whose are under the age of 20 and in males whose age 20 to 39 [1-5,27]. This disease is also the fifth leading cause of cancer-related deaths in females ages 20-39. This facts increase the importance of the researches on the tumor detection and this will present the opportunity for doctors to help save lives by detecting the disease earlier and perform necessary actions. Varieties of image processing techniques are available to be applied on various imaging modalities for tumor detection that will detect certain features of the tumors such as the shape, border, calcification and texture. These features will make the detection processes more accurate and easier as there are some standard characteristics of each features for a specific tumor [6-7].

All tumors will start small and grow with time. As they grow, they will become more conspicuous and increase the probability of showing their characters. A person with tumor usually has certain symptoms and this will bring that person to a physician. From this, they will 
be able to detect the smallest possible symptomatic malignant (cancerous) tumors that is in early stage and the smallest possible asymptomatic tumors in the screening process. Basically, there are many factors that can influence the appearance of tumors in different kind of processed images despite some common features of malignancies because of variation in the type of tissue and tumor. For large tumor, characteristic features often to be found while in small tumors, these features of malignancy do not appear to be many and some of them might represent themselves by secondary effects such as distortion in its architecture[8-12].

In the case of a suspected tumor, "a doctor may perform a neurologic exam to determine if the patient's senses, reflexes, mental status and memory are working normally. The doctor may also order imaging tests, including computed tomography (CT) or magnetic resonance imaging (MRI) of the brain, to pinpoint the tumor and show its size.’[13].

Imaging technology has progressed immensely in recent years. Different kind of images can be produced just by a single-click and different image processing techniques can be done to these images to study in detail about it. Machine learning and machine vision technology has also been used to solve numerous problems in medicine [20-22]. Image-based tumor detection uses one or more algorithms as the primary modeling. Some can detect edges, some can detect shapes while others can detect other features. With advances in camera sensing and computational technologies, advances in tumor detection using these features have been an extremely active research area in the intelligent medical community. Clearly, recent researches and trials have extremely help in advancing diagnostic tools for medical purposes but still, the fact that gains in survival need to be achieved by better diagnostic tools $[14,15]$.

\subsection{Magnetic Resonance Imaging (MRI)}

MRI is commonly used in the medical field for detection and visualization of details in the internal structure of the body. It is basically used to detect the differences in the body tissues which have a considerably better technique as compared to computed tomography [23]. Thus, this technique become a special technique especially for the brain tumor detection and cancer imaging [23]. Basically, for comparison, CT uses ionizing radiation while MRI uses strong magnetic field to align the nuclear magnetization that follows by changes the alignment of the magnetization by radio frequencies that can be detected by the scanner. The signal produced can be further processed later to gain extra information of the body [23].

This paper provide a review of image-based tumor detection. The authors then review imagebased tumor detection, commenting on techniques applied for color detection and shape detection. They provide their insights and perspectives on future research directions in imagebased tumor detection.

\section{BACKGROUND ON TUMOR DETECTION}

Nowadays, brain tumor has become one of the main cause for increasing mortality among children and adults [23]. Based on some researches, it has been found that the number of people suffering and dying from brain tumors has been increased to 300 per year during past few decades [23]. Figure 1 shows the incidence of brain tumor in various age groups. 


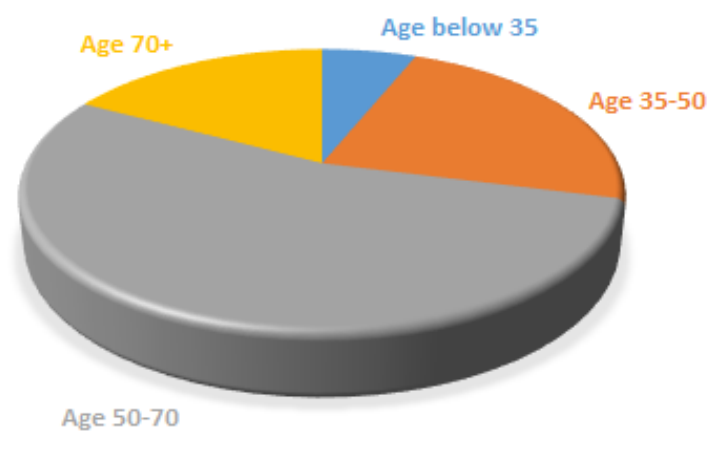

Figure 1: Brain tumor age distribution

Figure 2 shows the 5-year survival rates over the past three decades. It shows that the survival rates have improved with the advancement in imaging and diagnosis technology.

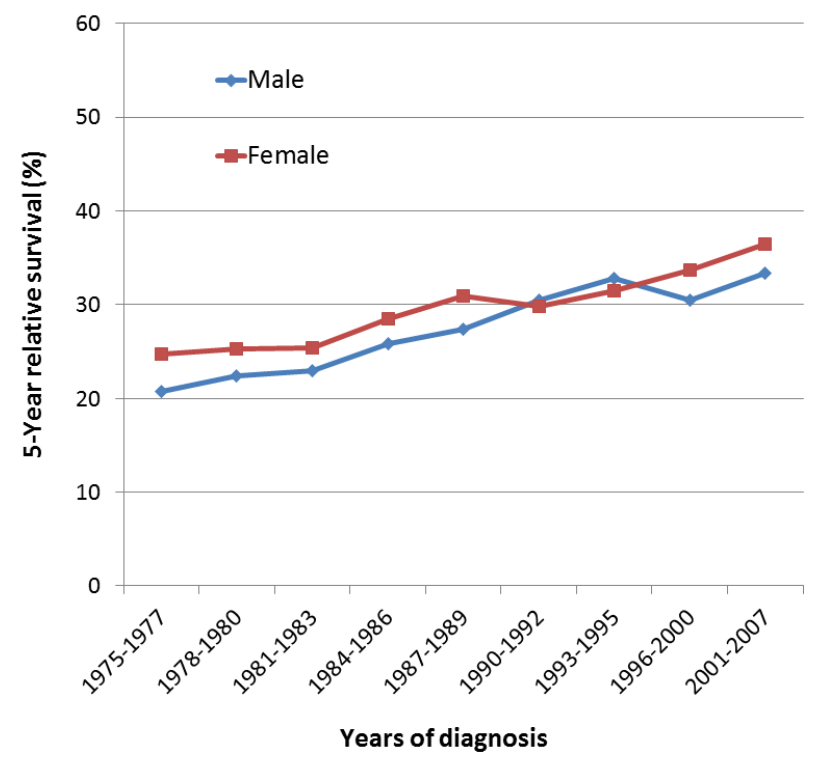

Figure 2: 5-Year brain tumor survival rate over years [24]

According to Nagalkar and Asole [16], CT-Scan technique usually used for monitoring the images of damaged brain part. The images of the CT Scans are shown in the form of gray scale images as the equipment for CT scans support this form of image color and for easy detection of tumor from the image [16]. For example, in the parietal section of the head scanned using CT scans, the Cerebrum part is shown in the form of the gray color while the veins and arteries parts in the form of creamish white color [16]. Any clotting that exist in the brain that show any kind of damage can be detected as dark gray in color. The process of extraction of parameters are basically like taking out per pixel information and then plotting it [16]. For an image obtained from CT-Scan, the images are shown in this manner; tumor appears white and brain damaged cells shown in black color, thus the binary values of the pixel showing the brain damaged cells are 0 and showing the tumor are 1, thus by extraction process, further analysis can be done such as checking and plotting in MATLAB [16]. The patient with damaged brain can be differentiated from normal patient by using this technique. In addition, tumor can also be detected clearly based on the image results [16]. 


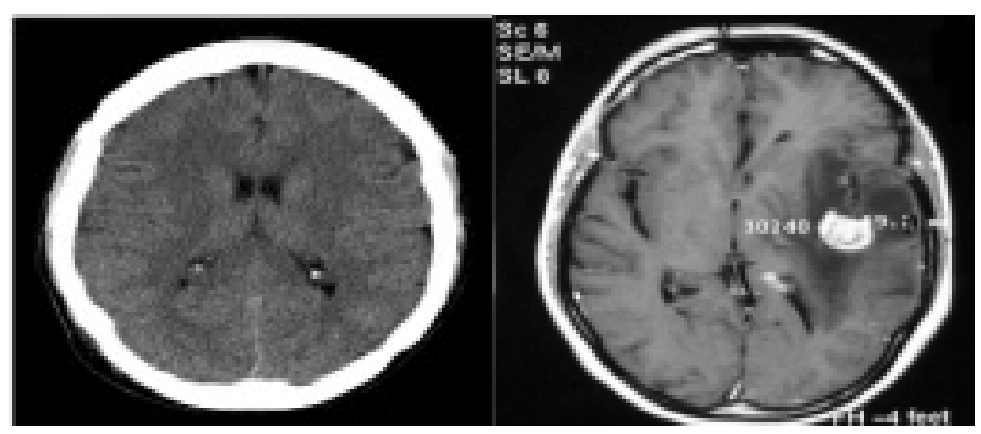

Figure 3: Brain CT Scan image a) Normal patient b) Tumor patient [16]

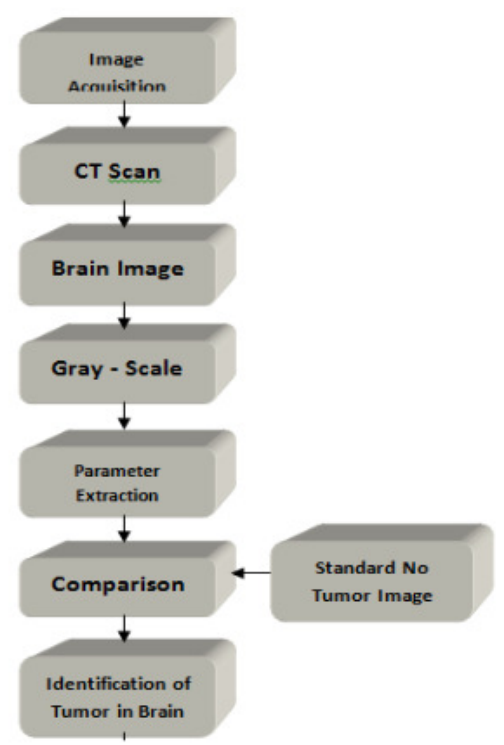

Figure 4: Basic flowchart to determine existence of tumor [16]

Additionally, this technique has been practiced to determine tumor patient's response to treatment since long time ago. The radiologist has made series of cross-sectional diameter measurements for indicator lesions purposes by using axial, incremental CT image data. Later, these measurements will be compared with the previous measurement scans [25]. Nevertheless, the measurement of lesion diameter does not represent the exact assessment of tumor size due to some factors, such as:

\section{Irregular lesions}

The lesions that grow other than sphere shape may not adequately represented by diameters' changes;

\section{Different measurement between inter-observer and intra-observer}

Referred to the image selection used for the measurement and the location of lesion boundary;

Different levels of scanning results collected from various diagnoses

The lesions may not be captured exactly at the same spot from one diagnosis to another. Hence, it affects the lesion's image in which causes comparisons between examinations becoming more difficult. 
On the other hand, with the ability to provide single breath-hold scans and overlapping reconstructions, the spiral CT is capable to improve the image lesions at reproducible levels from different diagnosis [25]. Even so, these lesions' measurements are still a subjective task and yet open for variability as described in previous factors (a) and (b). What follows is a summary of several approaches to the problems involved in nodule detection. Therefore, the researchers have divided these approaches into two groups of methods which are [25]:

(a) The process of detecting the pulmonary vasculature in order to help the detection of pulmonary nodules (if vasculature is successfully removed from the image, then only nodules should remain).

(b) The process of detecting objects in the CT image volume to distinguish between nodules and other structures.

\section{Proposed Methodology}

\subsection{Image Acquisition}

Images obtained or used should be of MRI scans and these scanned images are displayed in a two dimensional matrices which will have the number of pixels as its elements. Images are stored in Matlab and converted (if not already) to be displayed as a gray scale image of size 256*256 [23]. The size is important to reduce processing time or to be large enough to be considered for proper processing. The values of the gray scale image would range from 0 to 255 , where 0 represents total black color and 255 shows pure white color [23]. Anything in between shows a variety of values representing the intensities of gray color [18].

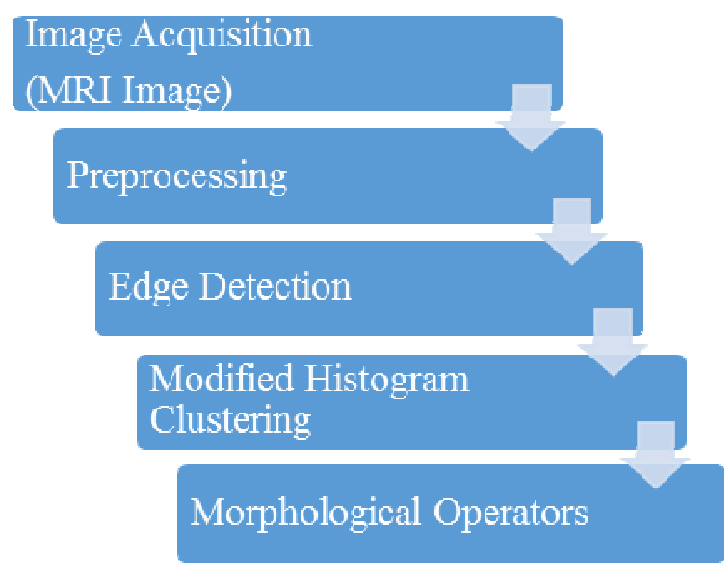

Figure 7: Block diagram of the Proposed Tumor Detection

\subsection{Pre-processing}

Pre-processing generally means removing noise and improving or altering image quality to suit a purpose. For this study, only commonly used enhancement and noise reduction techniques were implemented [23]. The image enhancement that the study is interested in should yield the result of more prominent edges and a sharpened image, noise will be reduced thus reducing the blurring or salt paper effect from the image that might produce errors [23].

After image enhancement, image segmentation will be applied. This step is vital as the improved and enhanced image will yield better results when detecting edges and improving the quality of 
the overall image. Edge detection will lead to determining and understanding the outline shape of the tumor. The following steps will be exemplified in the preprocessing stage [23]:

(a) Noise Removal: there is a wide range of filters available to be used to remove the noise from the images. Linear filters present on Matlab with simple line of code can also serve the purpose such as Gaussian and averaging filters. Salt and pepper noise is a common noise present in original captured images. Average filters for example, can remove these noise but with the sacrifice of sharpness of image. Median filter is also another example of a filter used to remove the noise like salt and pepper. In the median filter value of pixel is determined by the median of the values of its neighboring pixels [17]. This filter however, is less sensitive to the outliers [23].

(b) Any filter able to remove the noise present in the original image could be used for this purpose. However, in tumor detection, the sharpness of the edges, obtained from the sudden change of intensity, is a focal point and should be kept preserved. The next step will help improve the sharpness of the edges [23].

(c) Image Sharpening: sharpening is generally achieved by using high pass filters. After applying low pass filters (noise removing step), we now need to sharpen the image to ensure edges are kept. This is important as edges will detect and highlight the tumor for us. Gaussian filter (a high pass filter) is used to enhance the boundaries of the objects. It is widely used and the paper proposes to imitate [23].

\subsection{Edge Detection}

Edge detection is the most vital part in tumor detection. It is used to determine the boundaries of the object. In this step, canny edge filter is used.

"Five steps in canny filter: 1.Filters out noise in original image 2.Smoothing the image using filters 3.Finding the edge directions with library of orientation 4 . Relate to a direction that can be traced in an image 5.After edge directions are known, non-maximum suppression is done to thin out of remove edges based on threshold values determined." [18]

The two main purpose of using canny filter is [30]:

1. Control the amount of detail which appears in the edge image.

2. Suppress noise.

\subsection{Modified Histogram Clustering}

Histogram is one of the powerful techniques in image enhancement. The histogram of an image represents the relative frequency of occurrence of the various gray levels based on their individual values [28]. This is useful in stretching low-contrasting images. Clustering in which similar neighbored bins are grouped together and finally thresholding is set in order to detect the tumor.

One of the method in histogram clustering is the histogram values are plotted and the threshold value is fixed based on the gray level values and pixels in the image. To obtain this, we first plot the values and take a guess on the best value to use. The white parts of the image will have the maximum value of 255 and the black parts will translate to the value zero. Based on the values of the tumor, (certain studies keep first guess values to be at 128 as threshold value) the tumor image is processed. The values should be tested for various data sets of tumor images to identify the closest threshold value and the results are displayed later. Certain researches have specified that beyond the 132 mark, tumor is not detected clearly anymore [17]. Later, the tumor part is 
extracted via the morphological operators by taking and specifying the region of interest (ROI) and displaying it clearly.

\subsection{Morphological Operators}

Morphological operation involves dilation and erosion. Dilation combines two sets using vector addition. Erosion combines two sets using vector subtraction and is the dual operator of dilation [31]. Both are not invertible transforms.

Morphological techniques called "opening-by-reconstruction" and "closing-by-reconstruction" to "clean" up the image is used. These operations will create a gap between the high and low intensity objects and enhance it to locate images. The enhanced maxima inside each object that can be located using imregionalmax [26].

Opening is an erosion followed by a dilation, while opening-by-reconstruction is an erosion followed by a morphological reconstruction $[18,26]$.

\section{EXPERIMENTS}

The data is first presented according to example pictures of the image processes involved in the system. As discussed in the previous design part, the image used is of grayscale MRI brain scans. First we acquire the images and pre-processes it under a median filter. These are some of the examples the images:

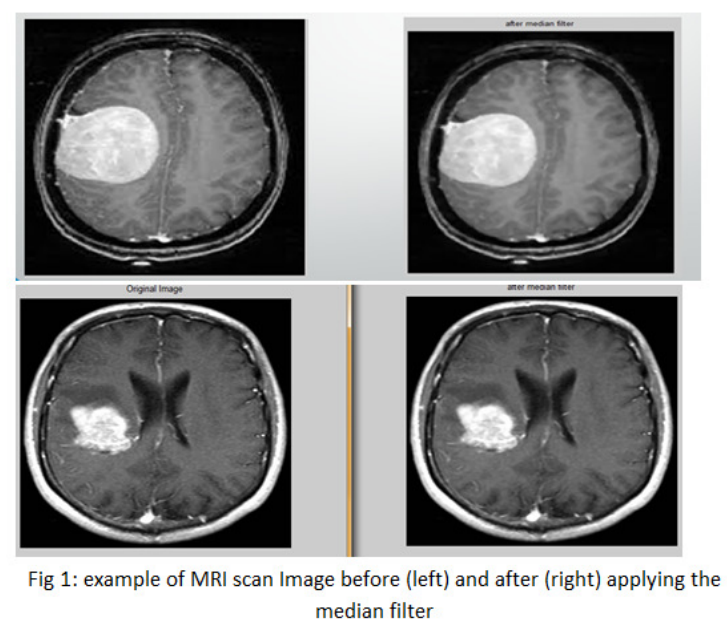

Figure 8: Images before (left) and after (right) median filter is applied

Next the edges of the images are specified with the sobel edge detection. The images processed are as follows: 
International Journal of Information Technology Convergence and Services (IJITCS) Vol.4, No.1, February 2014
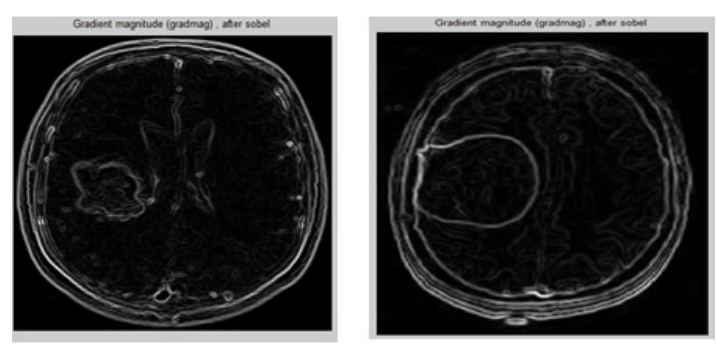

Figure 9: Images after sobel edge detection algorithm is applied

Later the images would go through a Modified Histogram Clustering - Color Threshold technique to identify the position of the tumor. According to various MRI scan images, the threshold values of the image's HSV for the tumor happens to be common and lies between these values:

Table 1. Threshold values applied

\begin{tabular}{|l|l|l|}
\hline HSV & High (\%) & Low (\%) \\
\hline Hue & 35 & 0 \\
\hline Saturation & 75 & 0 \\
\hline Value & 100 & 70 \\
\hline
\end{tabular}

The values are set as a threshold and applied with convolution with the original image to determine the location of the tumor.

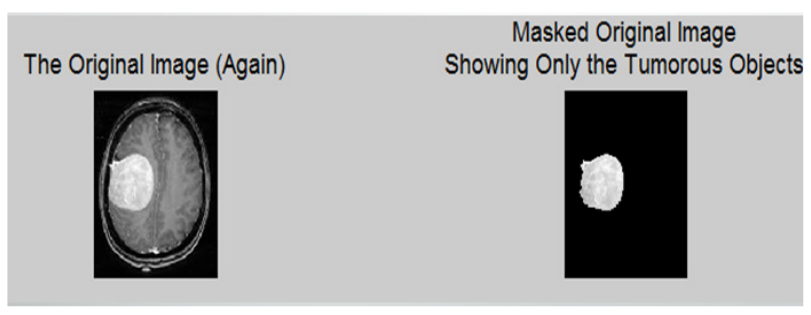

Figure 10: After applying modified threshold clustering technique

Through the syntax of bwlabel() on Matlab, each block of image found is subjected to a value of label. This allows us to gain property information about the block using the improps() syntax. The area can be found and displayed.

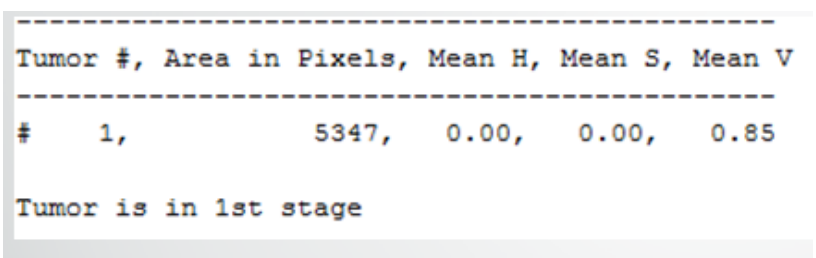

Figure 11: Display of results

Next we segment out the tumor subject from the original image. For this purpose, we use a watershed technique. The technique uses a series of algorithm that allows segmentation of high color contrast. The result of the imposed technique is as follows: 

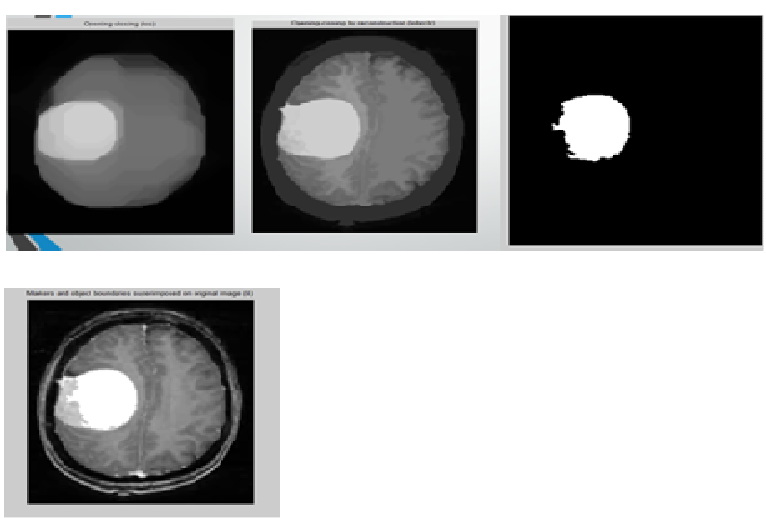

Figure 12: Watershed segmentation technique outcome

We used 50 neuroimages to optimize our system and 100 out-of-sample neuroimages to test our system. The proposed tumor detection and localization system was found to be able to accurately detect and localize brain tumor in magnetic resonance imaging. The proposed system achieved an error rate of $8 \%$ in identifying and localizing tumors.

We used a median filter for preprocessing because it yields a noise removed image that is still closely similar to the original image. In tumor detection, tampering too much with the intensity of color and contrast may lead to less accurate segmentation. This is because the tumor detection technique used in this study mainly focuses on the color contrast of tumors as its main feature.

According to previous studies, the median filter is also the most useful filter for MRI scan images because it deals best with salt and pepper noise without tampering too much with the color contrast.

In the color clustering technique, the intensity of the grey color contrast in tumor and the background brain sets the threshold value. This technique is used mainly because the color contrast is the only recognizable feature of a tumor. Even doctors rely solely on this feature to detect tumor from MRI scans.

Watershed segmentation is an important part of the project. It uses an opening-closing reconstruction technique which is basically the enhancing and dimming of foreground and background images. The contrast is then elevated. Reconstructing would highlight the high intensity objects. The reason for using this method is because tumor is detected according to grey color contrast or intensity. Watershed technique is suitable as it segments based on this feature.

According to the experiments and results obtained, the system records an $8 \%$ error. These errors are solely false positive errors. This means that images without tumors end up with detected blobs. The errors usually happen when the original images supplied are not grayscale images. The noise of the RGB or HSV conversion might have produced the error.

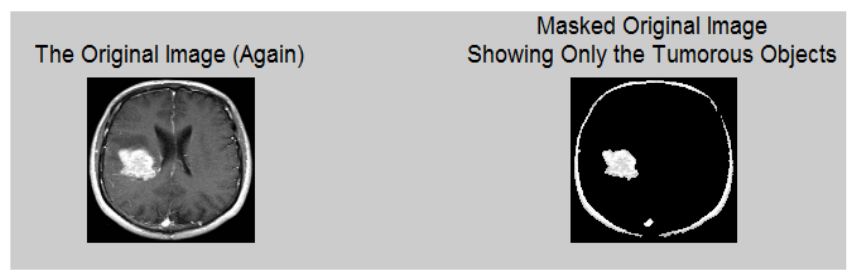

Figure 14: Error in modified threshold clustering 
The shows the detection of the skull as a form of tumor as well. Therefore it detects the patient to have a metastasis level tumor or a cancer of level 2 or above.

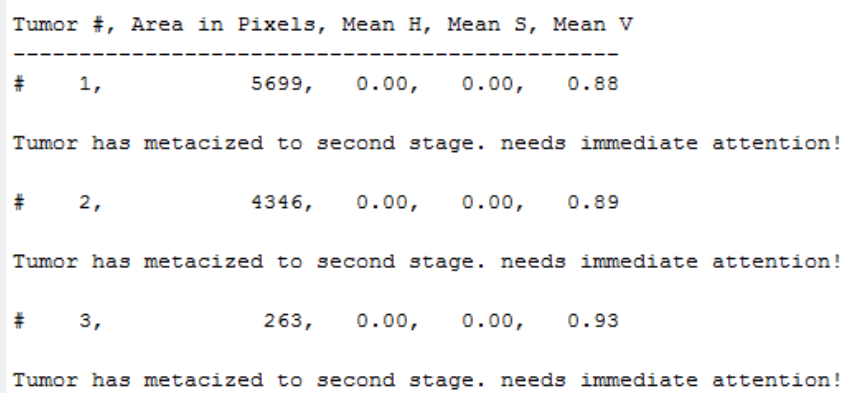

Figure 15: Error in detection

From the same error example as before, it shows that the program recognizes 3 areas of tumor. These areas are the example of the false positive errors recorded.

\section{CONCLUSION}

We propose an automatic brain tumor detection and localization framework that can detect and localize brain tumor in magnetic resonance imaging. The proposed brain tumor detection and localization framework comprises five steps: image acquisition, pre-processing, edge detection, modified histogram clustering and morphological operations. After morphological operations, tumors appear as pure white color on pure black backgrounds. We used 50 neuroimages to optimize our system and 100 out-of-sample neuroimages to test our system. The proposed tumor detection and localization system was found to be able to accurately detect and localize brain tumor in magnetic resonance imaging. This system achieved an error rate of $8 \%$. The preliminary results demonstrate how a simple machine learning classifier with a set of simple image-based features can result in high classification accuracy. The preliminary results also demonstrate the efficacy and efficiency of our five-step brain tumor detection and localization approach and motivate us to extend this framework to detect and localize a variety of other types of tumors in other types of medical imagery.

\section{REFERENCES}

[1] C. Croisille, M. Souto, M. Cova, S. Wood, Y. Afework, J.E. Kuhlman, E.A. Zerhouni. Pulmonary nodules: Improved detection with vascular segmentation and extraction with spiral CT. Radiology 197:397-401, 1995.

[2] T. Tozaki, Y. Kawata, N. Noki, H. Ohmatsu, K. Eguchi, N. Moriyama. Three-dimensional analysis of lung area using thin slice CT images. Medical Imaging Proc SPIE 2709:2-11, 1996.

[3] M.L. Giger, K.T. Bae, H. MacMahon. Computerized detection of pulmonary nodules in computed tomography images. Invest Radiol 29(4):459-465, 1994.

[4] S. Toshioka, K. Kanazawa, N. Niki, H. Satoh, H. Ohmatsu, K. Eguchi, N Moriyama. Computer aided diagnosis system for lung cancer based on helical CT images, image processing: KM Hanson, ed. Proc SPIE 3034:975-984, 1997.

[5] J. Toriwaki, A. Fukumura, T. Maruse. Fundamental properties of the gray weighted distance transformation, Trans IEICE Japan, J60-D(12):1101-1108, 1977.

[6] D. Cavouras, P. Prassopoulos, N. Pantelidis. Image analysis methods for the solitary pulmonary nodule characterization by computed tomography. Eur J Radiol 14: 169-172, 1992.

[7] M.F. McNitt-Gray, E.M. Hart, N. Wyckoff, J.W. Sayre, J.G. Goldin, D.R. Aberle. Characterization of solitary pulmonary nodules using features extracted from high resolution CT images. Radiology 205(P):395, 1997. 
[8] M.F. McNitt-Gray, E.M. Hart, N. Wyckoff, J.W. Sayre, J.G. Goldin, D. Aberle. The application of image analysis techniques to distinguish benign from malignant solitary pulmonary nodules imaged on CT. Proc SPIE Med Imag 3338, in press, 1998.

[9] Y. Kawata, N. Niki, H. Ohmatsu, R. Kakinuma. Classification of pulmonary nodules in thin section CT images based on shape characterization. IEEE Int Conf Image Proc 3:528-531, 1997.

[10] R.M. Summers, L.M. Pusanik, J.D. Malley. Automatic detection of endobronchial lesions with virtual bronchoscopy: comparison of two methods. Proc SPIE Med Imag 3338, in press, 1998.

[11] A.P. Reeves, B. Zhao, D.F. Yankelevitz, C.I. Henschke. Characterization of three-dimensional shape and size changes of pulmonary nodules over time from helical CT images. Radiology 205(P):396.

[12] D.F. Yankelevitz, E.R. Peters, B. Zhao, D Shaham, T Gade, CI Henschke. Can computer-aided morphologic analysis of solitary pulmonary nodules predict pathology? Radiology 205(P):529, 1997.

[13] Brain Tumors and Brain Cancer, Retrieved from: http://www.cedars-sinai.edu/Patients/HealthConditions/Brain-Tumors-and-Brain-Cancer.aspx, Retrieved on: 3 December 2013.

[14] American Brain Tumor Association. (2010). Facts and statistics, 2010. Retrieved from: http://www.abta.org/sitefiles/pdflibrary/ABTA-FactsandStatistics2010.pdf, Retrieved on: 3 December 2013.

[15] Central Brain Tumor Registry of the United States. (2010). CBTRUS statistical report: Primary brain and central nervous system tumors diagnosed in the United States in 2004-2006.Retrieved from: http://www.cbtrus.org/2010-NPCR-SEER/CBTRUS, Retrieved on: 3 December 2013.

[16] V.J. Nagalkar, S.S Asole, Brain Tumor Detection Using Digital Image Processing Based On Soft Computing. Journal Of Signal And Image Processing, Volume 3, Issue 3, pp.-102-105.

[17] D.A. Dahab, S.S.A. Ghoniemy, G.M. Selim, Automated Brain Tumor Detection and Identification Using Image Processing and Probabilistic Neural Network Techniques. International Journal of Image Processing and Visual Communication ISSN2319-1724 Volume 1, Issue 2, October 2012.

[18] H. Demuth and M. Beale, Neural Network Toolbox User's Guide, 2000

[19] T. Logeswari, M. Karnan, An improved implementation of brain tumor detection using segmentation based on soft computing, Journal of Cancer Research and Experimental Oncology Vol. 2(1), 2010, pp 6-14.

[20] Z.Z. Htike, S.L. Win Recognition of Promoters in DNA Sequences Using Weightily Averaged Onedependence Estimators, Procedia Computer Science, Volume 23, 2013, Pages 60-67, ISSN 18770509.

[21] Z.Z. Htike, S.L. Win Classification of Eukaryotic Splice-junction Genetic Sequences Using Averaged One-dependence Estimators with Subsumption Resolution, Procedia Computer Science, Volume 23, 2013, Pages 36-43, ISSN 1877-0509.

[22] Z.Z. Htike, S. Egerton, Y.C. Kuang, A Monocular View-Invariant Fall Detection System for the Elderly in Assisted Home Environments, 7th International Conference on Intelligent Environments (IE), 2011, vol., no., pp.40,46, 25-28 July 2011.

[23] A. Mustaqeem, A. Javed, T. Fatima, An Efficient Brain Tumor Detection Algorithm Using Watershed \& Thresholding Based Segmentation International Journal of Image, Graphics and Signal Processing, Vol.4, No.10, 2012, pp34-39.

[24] Global cancer statistics: Brain tumor, Retrieved from: http://www.medinfographics.com/cancerstatistics/brain-tumor/, Retrieved on: 5 December 2013.

[25] M.F. McNitt-Gray, M.S. Brown X-Ray and CT, Image-Processing Techniques for Tumor Detection, Edited by R.N. Strickland, CRC Press 2002.

[26] Marker-Controlled Watershed Segmentation, Retrieved http://www.mathworks.com/products/demos/image/watershed/ipexwatershed.html, Retrieved on: 6 December 2013.

[27] Brain Tumor Information, Retrieved from: http://www.abta.org/brain-tumor-information/ Retrieved on: 6 December 2013.

[28] T. Acharya, A.K. Ray, Image Processing: Principles and Applications, Wiley-Interscience, 2005.

[29] S. Xavierarockiaraj, K. Nithya, R.M. Devi Brain Tumor Detection Using Modified Histogram Thresholding-QuadrantApproach, Journal of Computer Applications, Volume V, Issue 1, 2012.

[30] J. Omwoyo, Canny edge detector, Seminar, Lappeenranta University of Technology, 2007.

[31] N. Liu, M. Lu, Computational Geometry, Graphs and Applications, Lecture Notes in Computer Science, 2010. 